\title{
Histone methyltransferase Smyd3 regulates early embryonic lineage commitment in mice
}

\author{
Shinnosuke Suzuki ${ }^{*}$, Yusuke Nozawa*, Satoshi Tsukamoto ${ }^{1}$, Takehito Kaneko ${ }^{2}$, Hiroshi Imai \\ and Naojiro Minami \\ Laboratory of Reproductive Biology, Graduate School of Agriculture, Kyoto University, Kyoto 606-8052, Japan, \\ ${ }^{1}$ Laboratory of Animal and Genome Sciences Section, National Institute of Radiological Sciences, Chiba 263-8555, \\ Japan and ${ }^{2}$ Graduate School of Medicine, Institute of Laboratory Animals, Kyoto University, Kyoto 606-8501, Japan \\ Correspondence should be addressed to N Minami; Email: oog1nao@kais.kyoto-u.ac.jp
}

*(S Suzuki and Y Nozawa contributed equally to this work)

\begin{abstract}
SET and MYND domain-containing protein 3 (Smyd3) is a histone H3 lysine 4 (H3K4) di- and tri-methyltransferase that forms a transcriptional complex with RNA polymerase II and activates the transcription of oncogenes and cell cycle genes in human cancer cells. However, the study of Smyd3 in mammalian early embryonic development has not yet been addressed. In the present study, we investigated the expression pattern of Smyd3 in mouse preimplantation embryos and the effects of RNA interference (RNAi)-mediated Smyd3 repression on the development of mouse embryos. We showed that Smyd3 mRNA levels increased after the two-cell stage, peaked at the four-cell stage, and gradually decreased thereafter. Moreover, in two-cell to eight-cell embryos, SMYD3 staining was more intense in the nuclei than it was in the cytoplasm. In Smyd3-knockdown embryos, the percentage of inner cell mass (ICM)-derived colony formation and trophectoderm (TE)-derived cell attachment were significantly decreased, which resulted in a reduction in the number of viable offspring. Furthermore, the expression of $\mathrm{Oct} 4$ and $\mathrm{Cdx} 2$ during mid-preimplantation gene activation was significantly decreased in Smyd3-knockdown embryos. In addition, the transcription levels of ICM and epiblast markers, such as Oct4, Nanog, and Sox2, the transcription levels of primitive endoderm markers, such as Gata6, and the transcription levels of TE markers, such as Cdx2 and Eomes, were significantly decreased in Smyd3-knockdown blastocysts. These findings indicate that SMYD3 plays an important role in early embryonic lineage commitment and peri-implantation development through the activation of lineage-specific genes.

Reproduction (2015) 150 21-30
\end{abstract}

\section{Introduction}

Embryonic development in mammals is characterized by an initial preimplantation phase that serves to prepare the embryo for implantation. Transcription from the newly formed zygotic genome, known as zygotic gene activation $(Z G A)$, begins after fertilization between the late one-cell stage and the two-cell stage (Schultz \& Worrad 1995, Latham \& Schultz 2001, Li et al. 2010). Subsequently, mid-preimplantation gene activation (MGA) occurs during the four- to eight-cell stages (Hamatani et al. 2004). Both ZGA and MGA consist of new gene expression from the embryonic genome, and both steps require proper lineage commitment and differentiation. The first lineage differentiation gives rise to the inner cell mass (ICM) and trophectoderm (TE). The pluripotency of the ICM lineage is regulated by the transcription factors Oct4 (also known as Pou5f1), Nanog, and Sox2 (Nichols et al. 1998, Avilion et al. 2003, Mitsui et al. 2003), and the specification and differentiation of the TE lineage is regulated by the transcription factors $C d x 2$ and Eomes (Russ et al. 2000, Strumpf et al. 2005). Before implantation, the ICM gives rise to the epiblast (EPI), which predominantly expresses Nanog, and the primitive endoderm $(\mathrm{PE})$, which predominantly expresses Gata6 (Rossant 2004, Chazaud et al. 2006). The EPI eventually gives rise to the fetus, whereas the PE develops into the visceral and parietal endoderm of the yolk sacs and the TE becomes the fetal placenta.

In general, gene expression is regulated through the transition of several epigenetic factors, including transcription factors, chromatin-remodeling factors, and some enzymes. Examples of the epigenetic changes that take place during preimplantation development include DNA methylation, histone post-translational modifications, and histone variant exchange (Sarmento et al. 2004, Santos et al. 2005, Hirasawa et al. 2008, Akiyama et al. 2011). Drastic changes in many varieties 
of histone post-translational modifications occur during ZGA. Histone post-translational modifications are introduced in a variety of ways. Several enzymes contribute to histone methylation (Zhang \& Reinberg 2001), acetylation (Sterner \& Berger 2000), phosphorylation (Nowak \& Corces 2004), and ubiquitination (Shilatifard 2006). With respect to methylation, modifications of lysines 4, 36, and 79 of histone $\mathrm{H} 3$ (referred to as H3K4, $\mathrm{H} 3 \mathrm{~K} 36$, and H3K79 respectively) are associated with transcriptional activation, whereas modifications of lysines 9 and 27 of histone $\mathrm{H} 3$ and lysine 20 of histone $\mathrm{H} 4$ (referred to as $\mathrm{H} 3 \mathrm{~K} 9, \mathrm{H} 3 \mathrm{~K} 27$, and $\mathrm{H} 4 \mathrm{~K} 20$ respectively) are associated with transcriptional repression (Lepikhov \& Walter 2004, Sarmento et al. 2004). Except for the H3K79 methyltransferase, which is known as DOT1L, histone methyltransferases (HMTase) include a conserved catalytic domain called the SET domain (Zhang \& Reinberg 2001, Feng et al. 2002). SET and MYND domain containing protein 3 (Smyd3) encodes a protein comprising 428 amino acids and containing a SET domain, a MYND-type zinc-finger domain, and a SET-N region. SMYD3 has been reported to be capable of methylating both $\mathrm{H} 3 \mathrm{~K} 4$ and $\mathrm{H} 4 \mathrm{~K} 5$ (Hamamoto et al. 2004, Van Aller et al. 2012). Evidence has accumulated that suggests that SMYD3 recruits RNA polymerase II through an RNA helicase to form a transcription complex and that it elicits its oncogenic effects by activating the transcription of downstream target genes (Hamamoto et al. 2004, 2006, Liu et al. 2007, 2013). Previous reports have demonstrated that enhanced expression of SMYD3 is essential for the growth of human cancer cells (Hamamoto et al. 2004, 2006), whereas the suppression of SMYD3 expression leads to apoptosis and the inhibition of cell growth, migration, and invasion (Chen et al. 2007, Zou et al. 2009). Recent studies have determined that Smyd3 plays an important role in the development of heart and skeletal muscle during zebrafish embryogenesis (Fujii et al. 2011). However, the role of SMYD3 in mammalian early embryonic development has not been previously addressed.

In the present study, we examined the expression pattern of Smyd3 mRNA and the localization of SMYD3 protein in mouse preimplantation embryos, and we found that siRNA-mediated knockdown of Smyd3 during the early stages of embryonic development suppressed Oct4 and $C d x 2$ at MGA. Additionally, Smyd3 knockdown early in development reduced the blastocyst-stage expression of ICM/EPI markers (e.g., Oct4, Nanog, and Sox2), PE markers (e.g., Gata6), and TE markers (e.g., Cdx2 and Eomes). However, the number of apoptotic cells was not increased until day 4 after the outgrowth experiments (E7.5) in Smyd3knockdown embryos. From these results, we propose that SMYD3 plays an important role in early embryonic lineage commitment through the activation of lineagespecific genes.

\section{Materials and methods}

\section{IVF and embryo culture}

IVF was performed as previously described (Suzuki et al. 2015). Three hours after insemination, fertilized eggs were washed and cultured in K-modified simplex optimized medium (KSOM) containing $4 \mathrm{mg} / \mathrm{ml}$ BSA under mineral oil (SigmaAldrich) at $37^{\circ} \mathrm{C}$, in an atmosphere of $5 \% \mathrm{CO}_{2}$ in air until embryonic day 5.5 (E5.5) or used for microinjection (Tsukamoto et al. 2013) and cultured until embryonic day 5.5 (E5.5).

\section{RNA extraction and quantitative RT-PCR}

Metaphase II oocytes, and one-cell, two-cell, four-cell, eightcell, morula, and blastocyst stage embryos were collected at 14, 28, 50, 62, 69, 90 and $122 \mathrm{~h}$ after the hCG injection respectively. RNA extraction and quantitative RT-PCR (qRTPCR) were performed as previously described (Suzuki et al. 2013). Total RNA from 30 embryos at each stage was extracted using the TRIzol reagent (Invitrogen). Transcription levels were determined using three different sets of 30 embryos per stage and normalized to $H 2 a f z$, which is known as a stable reference gene for the normalization of gene expression in mouse preimplantation embryos (Jeong et al. 2005, Mamo et al. 2007), or Gapdh; relative gene expression was analyzed using the $2^{-\Delta \Delta C t}$ method (Livak \& Schmittgen 2001). All primers used for PCR are listed in Supplementary Table 1, see section on supplementary data given at the end of this article.

\section{Microinjection of siRNA}

Fertilized embryos transferred to KSOM were microinjected into the cytoplasm with 5-10 pl of $100 \mu \mathrm{M} S m y d 3$ siRNA (siSmyd3-1: 5'-GCAGGGUUAUCGUCAAGCUGA-3'; siSmyd3-2: 5'-GUCGUGGCGUAGUCUGUGAUC-3'; RNAi, Inc., Tokyo, Japan) between 3 and $4 \mathrm{~h}$ after insemination. The same amount of negative control siRNA (siControl; RNAi, Inc.), which contained scrambled sequences from the siSmyd3-1 or siSmyd3-2 construct, was also microinjected as a control. To examine the developmental competency and hatching ability, embryos were observed at 50 (E1.5), 74 (E2.5), 98 (E3.5), 122 (E4.5) and $146 \mathrm{~h}$ (E5.5) after the hCG injection. After the siRNA injection, embryos were harvested at either the four- and eightcell stages (62 and $69 \mathrm{~h}$ after the hCG injection respectively) for qRT-PCR and immunostaining, or at the morula/blastocyst stage (98 $\mathrm{h}$ after the hCG injection) for outgrowth experiments, or at the blastocyst stage (122 h after the hCG injection) for qRT-PCR, immunostaining, and immunoblotting.

\section{Outgrowth experiment and embryo transfer}

Outgrowth experiments were performed using morula or blastocyst stage embryos collected $98 \mathrm{~h}$ after the hCG injection as previously described (Yamada et al. 2010). After culture for 4 days, the percentage of blastocysts that underwent outgrowth was calculated and photographed. A portion of the embryos that reached the two-cell stage after microinjection was transferred into the oviducts of $0.5 \mathrm{dpc}$ pseudo-pregnant ICR 
female mice. These females were killed on day 19, and the pups were counted. The experiment was repeated four times.

\section{Immunostaining}

Embryos for immunostaining were collected as described earlier in the Materials and methods. For SMYD3, trimethylated H3K4 (H3K4me3), and EOMES staining, embryos were fixed in $4 \%$ paraformaldehyde in PBS for $20 \mathrm{~min}$ at $4{ }^{\circ} \mathrm{C}$ after the removal of the zona pellucidae with acidic Tyrode's solution $(\mathrm{pH} 2.5)$. After being washed three times in PBS containing $0.3 \%$ polyvinylpyrrolidone (PVP K-30, Nacalai Tesque, Kyoto, Japan) (PBS/PVP), fixed embryos were treated with $0.5 \%$ Triton X-100 (Sigma-Aldrich) in PBS for $40 \mathrm{~min}$ at room temperature (RT) and were blocked in PBS containing 1\% BSA for $1 \mathrm{~h}$ at RT (for SMYD3 and EOMES) or $3 \%$ BSA overnight at $4{ }^{\circ} \mathrm{C}$ (for $\mathrm{H} 3 \mathrm{~K} 4 \mathrm{me} 3)$. Next, embryos were either incubated overnight at $4{ }^{\circ} \mathrm{C}$ with a rabbit anti-SMYD3 antibody (1:100 dilution, $10 \mu \mathrm{g} / \mathrm{ml}$; ab16027, Abcam Ltd, Cambridge, UK) or a rabbit anti-EOMES antibody (1:500 dilution, $0.4 \mu \mathrm{g} / \mathrm{ml}$; ab23345, Abcam Ltd) or incubated for $1 \mathrm{~h}$ at RT with a rabbit antiH3K4me3 antibody (1:200 dilution, $2.5 \mu \mathrm{g} / \mathrm{ml}$; ab8580, Abcam Ltd) in antibody dilution buffer (PBS containing 1\% BSA). Embryos were washed three times in antibody dilution buffer and then incubated with the appropriate secondary antibody diluted at 1:500 (Alexa Fluor 488-conjugated goat anti-rabbit IgG or Alexa Fluor 594-conjugated goat anti-rabbit IgG, Invitrogen) for $1 \mathrm{~h}$ at RT. Immunostaining with normal rabbit IgG (sc-2027, Santa Cruz Biotechnology) was performed as a negative control for the specificity of the anti-SMYD3 antibody. For NANOG, SOX2, and GATA6 staining, embryos were fixed, permeabilized, and blocked as previously described (Ralston \& Rossant 2008). Next, embryos were incubated overnight at $4{ }^{\circ} \mathrm{C}$ with a rabbit anti-NANOG antibody (1:1000 dilution, $1 \mu \mathrm{g} / \mathrm{ml}$; ab5731, Millipore, Bedford, MA, USA), a goat anti-SOX2 antibody (1:100 dilution, $2 \mu \mathrm{g} / \mathrm{ml}$; sc-17320, Santa Cruz Biotechnology), or a goat antiGATA6 antibody (1:1000 dilution, $0.2 \mu \mathrm{g} / \mathrm{ml}$; AF1700, R\&D Systems, Inc., Minneapolis, MN, USA) in blocking solution overnight at $4{ }^{\circ} \mathrm{C}$. Embryos were washed three times in blocking solution and then incubated with the appropriate secondary antibody diluted at 1:750 (Alexa Fluor 594-conjugated goat anti-rabbit IgG, Invitrogen) or 1:500 or 1:750 (Alexa Fluor 594-conjugated rabbit anti-goat IgG, Invitrogen) for $1 \mathrm{~h}$ at RT. After staining, the samples were washed three times in antibody dilution buffer or blocking solution for $15 \mathrm{~min}$, and nuclei were stained in PBS containing $10 \mu \mathrm{g} / \mathrm{ml}$ Hoechst 33342 (Sigma-Aldrich) for $10 \mathrm{~min}$ at RT. Immunofluorescent staining for OCT4 and CDX2 was performed as previously described (Isaji et al. 2013). After staining, embryos were mounted on slides in 50\% glycerol/ PBS, and SMYD3-related signals were observed using a fluorescence microscope (BZ-X700, Keyence, Osaka, Japan) equipped with structured illumination microscopy (Gustafsson 2005, Hosny et al. 2013). Fluorescein signals related to OCT4, CDX2, H3K4me3, NANOG, SOX2, GATA6, and EOMES were detected using fluorescence microscopy (BX50, Olympus). At least 20 samples were examined in each group. The numbers of ICM and TE cells were determined by counting OCT4- and
CDX2-positive cells respectively. In order to count the number of cells and observe the localization of OCT4, CDX2, NANOG, SOX2, GATA6, and EOMES in Smyd3knockdown embryos, exposure time was extended as compared to that of control embryos. The total embryonic cell numbers were obtained by adding the numbers determined for the ICM and TE cells.

\section{Immunoblotting}

Immunoblotting was performed as previously described (Suzuki et al. 2013). Total proteins from 100 embryos were extracted using SDS sample buffer/lysis buffer (1:1). Primary antibody was used; a mouse anti- $\alpha$-TUBULIN antibody (1:5000 dilution, $1.16 \mu \mathrm{g} / \mathrm{ml}$; T9026; Sigma-Aldrich), a rabbit anti-SMYD3 antibody (1:100 dilution, $10 \mu \mathrm{g} / \mathrm{ml})$, a rabbit antiH3K4me3 antibody (1:200 dilution, $2.5 \mu \mathrm{g} / \mathrm{ml})$, a rabbit anti-OCT4 antibody (1:500 dilution, $400 \mathrm{ng} / \mathrm{ml} ; \mathrm{C}-10$; sc-5279, Santa Cruz Biotechnology), or a mouse anti-CDX2 antibody (1:500 dilution, $100 \mu \mathrm{g} / \mathrm{ml}$; CDX-88; BioGenex, San Ramon, CA, USA). The following secondary antibodies were used: an HRP-conjugated anti-mouse secondary antibody (1:2000 or 1:10 000 dilution; GE Healthcare UK Ltd, Little Chalfont, UK) or an HRP-conjugated anti-rabbit secondary antibody (1:1000 or 1:2000 dilution; GE Healthcare UK Ltd) in TBS-T for $1 \mathrm{~h}$ at RT. The membrane was extensively washed three times with TBS-T, and then bound antibodies were detected using the ECL system (GE Healthcare UK Ltd). $\alpha$-TUBULIN was used as an internal control.

\section{TUNEL assay}

Apoptotic cells in embryos at E3.5, E4.5, and E5.5 and outgrowth embryos at E6.5 and E7.5 were identified with the In Situ Cell Death Detection kit (Roche Diagnostics Corp.) using the protocol recommended by the manufacturer.

\section{Statistical analysis}

All data are expressed as means \pm s.E.M. Statistical analysis of the data was performed by ANOVA with Student's $t$-test for comparing two groups.

\section{Ethical approval for the use of animals}

All animal experiments were approved by the Animal Research Committee of Kyoto University (Permit no. 24-17) and were performed in accordance with the committee's guidelines.

\section{Results \\ The expression of the Smyd3 $m R N A$ and protein in mouse preimplantation embryos}

First, we revealed the expression pattern of Smyd3 mRNA and the localization of SMYD3 protein in mouse preimplantation embryos. qRT-PCR analysis of Smyd3 mRNA in preimplantation embryos indicated that the 
expression levels increased after the one-cell stage, peaked at the four-cell stage, and then slightly decreased until the blastocyst stage (Fig. 1A). Furthermore, immunostaining showed that SMYD3 dominantly localized to the nuclei from the two-cell to the eight-cell stage (Fig. 1B).

\section{Effects of Smyd3 knockdown on the development of mouse embryos}

In order to investigate the role of Smyd3 in early embryonic development, we knocked down the expression of Smyd3 in mouse preimplantation embryos. Embryos injected with siRNA that targeted Smyd3 (siSmyd3-1 and siSmyd3-2) were cultured until E5.5. qRT-PCR and immunoblotting showed that the reduction

A
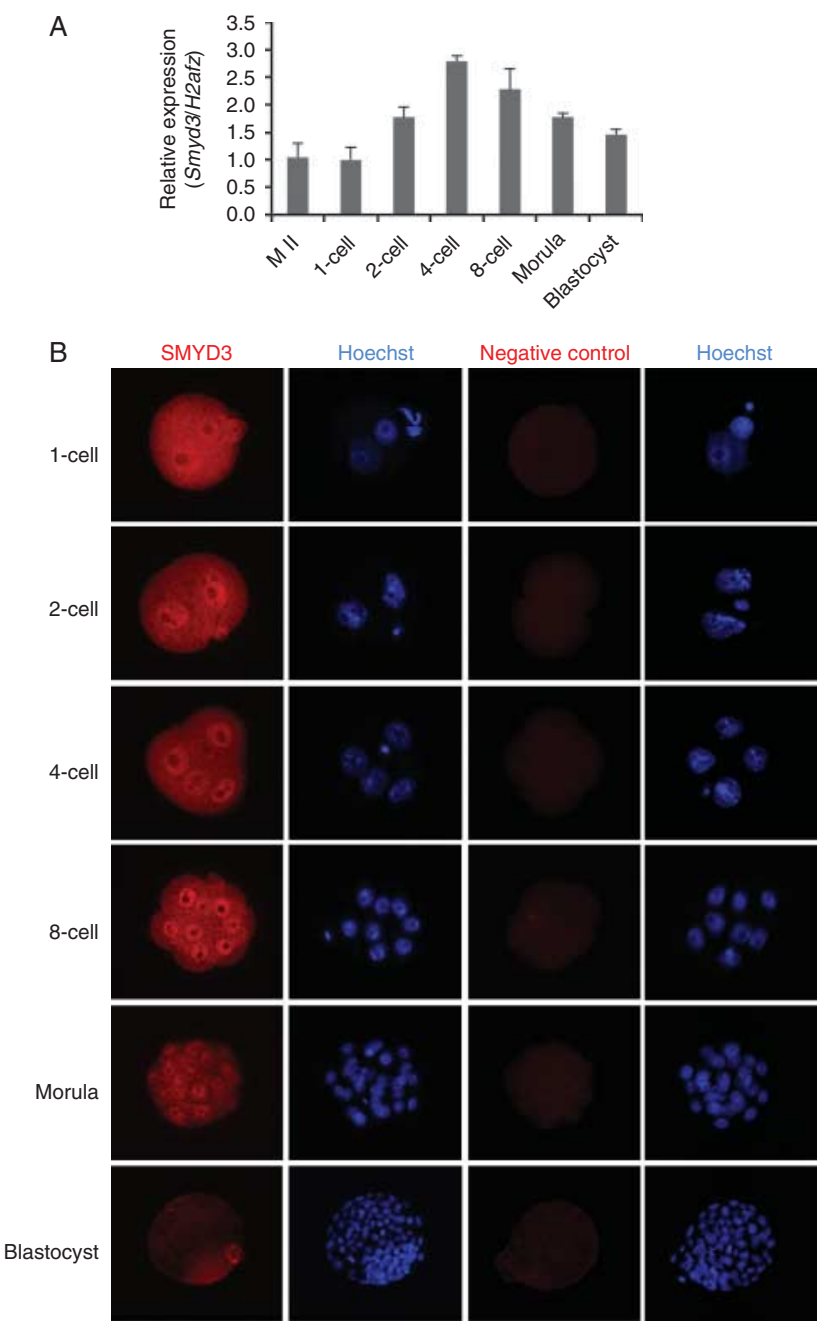

Figure 1 Smyd3 expression and the localization of SMYD3 in preimplantation embryos. (A) qRT-PCR analysis of Smyd3 expression in preimplantation embryos. The expression levels at each developmental stage were normalized using $\mathrm{H} 2 \mathrm{afz}$ as an internal control. Data are expressed as means \pm S.E.M. $(n=3)$. (B) Localization of Smyd3 in mouse preimplantation embryos. SMYD3 (red) is detected by immunofluorescence and nuclei (blue) are stained with Hoechst dye. in the expression of Smyd3 mRNA and protein was observed at the blastocyst stage (Fig. 2A and B; Supplementary Figure 1A, see section on supplementary data given at the end of this article). Additionally, immunostaining showed that SMYD3 protein levels were also reduced beginning in the one-cell stage and continuing through the blastocyst stage (Fig. 2C). Nevertheless, no differences between Smyd3-knockdown and control embryos were noted with respect to morphology or the percentage of embryonic development up to E5.5 (Fig. 3A and B; Supplementary Figure $1 \mathrm{~B}$ and C). Cell numbers in $\mathrm{E} 4.5$ blastocysts were counted after OCT4 (ICM) and CDX2 (TE) staining. The data demonstrated that Smyd3-knockdown blastocysts had normal numbers of cells in both populations (Fig. 3C). To examine the pluripotency of Smyd3-knockdown embryos, outgrowth experiments were performed on E3.5 embryos. In control embryos, the percentages of successful attachment and ICM-derived colony formation were 88.9 and $81.1 \%$ respectively, whereas in Smyd3-knockdown embryos, the percentages were 71.4 and $45.8 \%$ respectively (Fig. 3D and E). In addition, to test the viability of Smyd3-knockdown embryos in vivo, siSmyd3-1-injected embryos were transferred into the oviducts of pseudo-pregnant mice. The percentage of offspring derived from Smyd3-knockdown embryos was significantly reduced as compared to controls (Table 1 ).

\section{Knockdown of Smyd3 leads to the down-regulation of linage-specific genes}

In order to analyze the reasons for the defect of periimplantation embryonic development, we performed qRT-PCR on four- and eight-cell stage embryos for Smyd3 and early differentiation markers, such as Oct4 and $C d \times 2$. In addition, we performed immunostaining to confirm the levels of H3K4me3 in Smyd3-knockdown embryos at the four- and eight-cell stages. The results demonstrated that the expression of Smyd3 mRNA was significantly decreased in Smyd3-knockdown embryos (Fig. 4A); however, H3K4me3 levels remained unchanged (Fig. 4B). Interestingly, the transcription of Oct4 at the four-cell and eight-cell stages and that of $C d \times 2$ at the eight-cell stage was significantly decreased in Smyd3-knockdown embryos (Fig. 4C and D). To further investigate the influence of the reduced expression of Smyd3, Oct4, and Cdx2 at the four- and eight-cell stages, we performed qRT-PCR on E4.5 blastocyst stage embryos for $\mathrm{H} 2 \mathrm{afz}$ - an internal control - and lineage-specific genes, such as the ICM/EPI markers Oct4, Nanog, and Sox2; the PE marker Gata6; and the TE markers $C d x 2$ and Eomes. The results demonstrated that the transcription levels of Oct4, Nanog, Sox2, Gata6, Cdx2, and Eomes were all significantly decreased in Smyd3-knockdown embryos at the blastocyst stage, whereas $\mathrm{H} 2 \mathrm{afz}$ transcript 


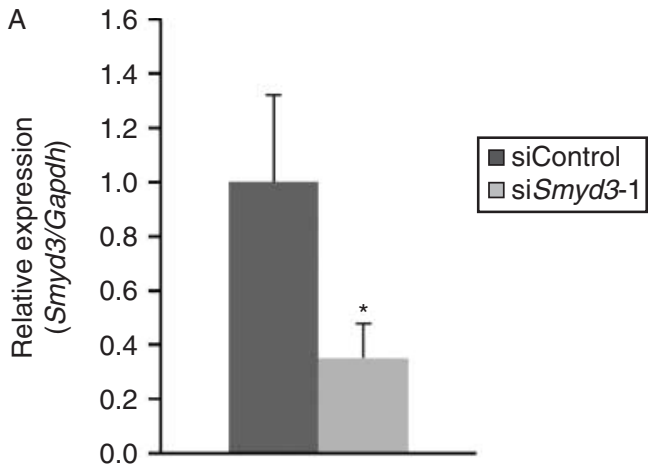

B
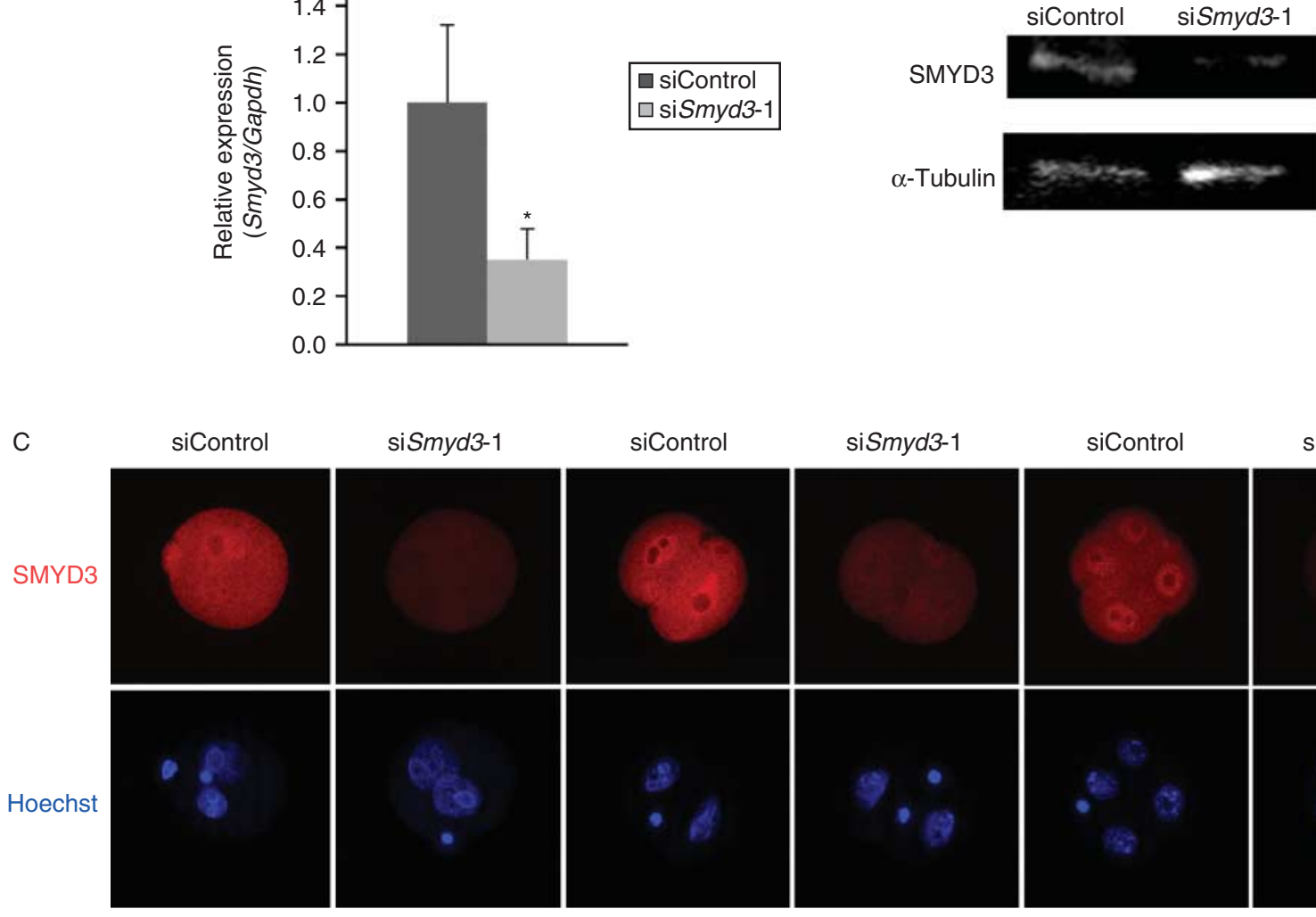

1 -cell

siControl

siSmyd3-1

siControl

siSmyd3-1

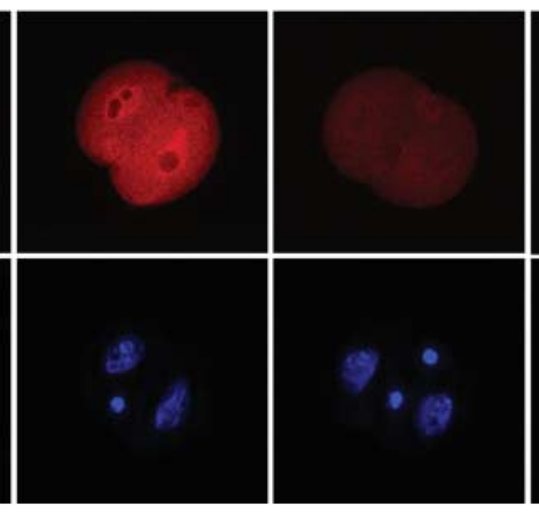

2-cell

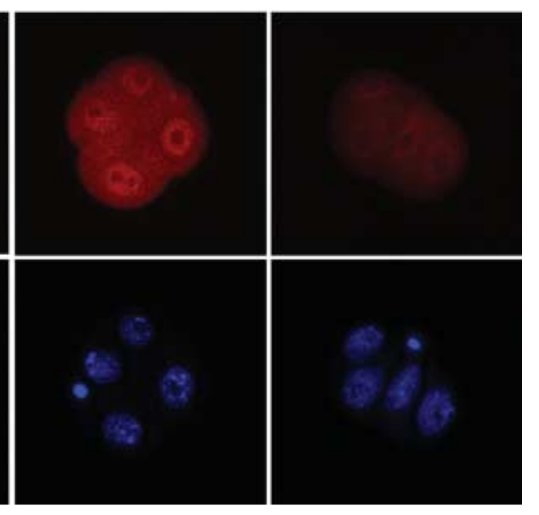

4-cell

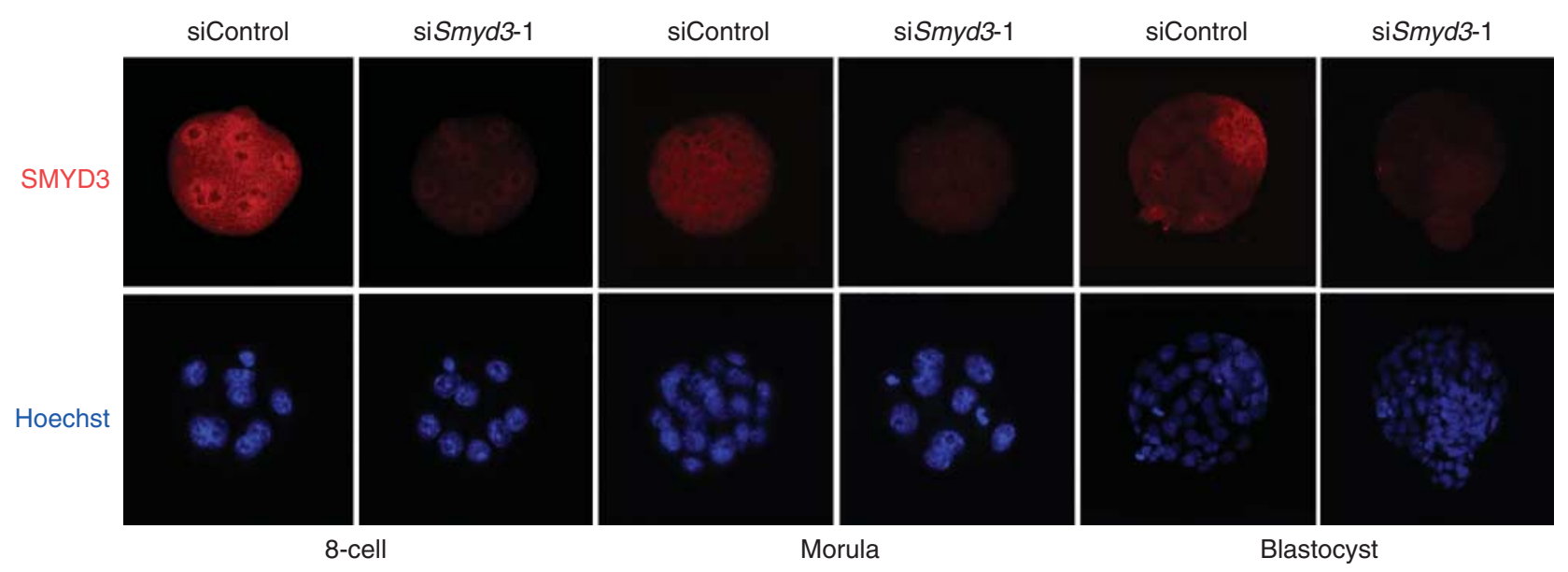

Figure 2 Reduction in the expression of Smyd3 mRNA and protein at the blastocyst stage. (A) qRT-PCR analysis of Smyd3 mRNA at the blastocyst stage in Smyd3-knockdown and control embryos $\left({ }^{*} P<0.05\right)$. The expression levels were normalized using Gapdh as an internal control. Data are expressed as means \pm s.E.M. $(n=3)$. (B) Immunoblot analysis of SMYD3 and $\alpha$-TUBULIN in Smyd3-knockdown and control blastocyst embryos. (C) Immunostaining of SMYD3 protein in Smyd3-knockdown and control blastocyst embryos (red, SMYD3; blue, chromatin).

levels remained unchanged (Fig. 5A; Supplementary Figure 2A). Furthermore, the protein levels of OCT4, CDX2, NANOG, SOX2, GATA6, and EOMES were confirmed on E4.5 blastocysts. The results demonstrated that the levels of OCT4, CDX2, NANOG, SOX2, GATA6, and EOMES were significantly reduced in
Smyd3-knockdown embryos at the blastocyst stage (Fig. 5B; Supplementary Figures 2B and 3A, see section on supplementary data given at the end of this article). Additionally, global H3K4me3 levels also remained unchanged in the treated embryos at the blastocyst stage (Supplementary Figure 3B). 
A

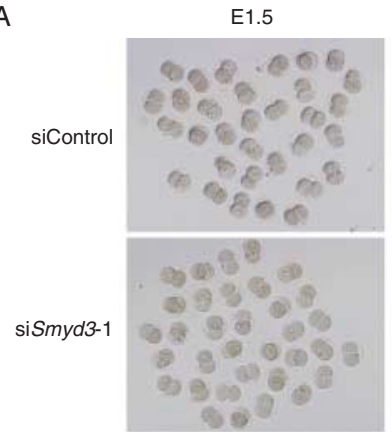

E2.5

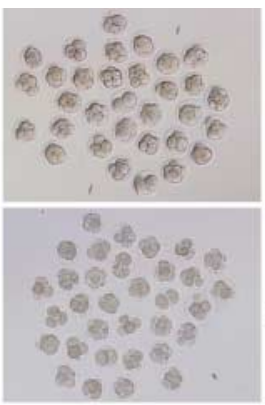

E3.5

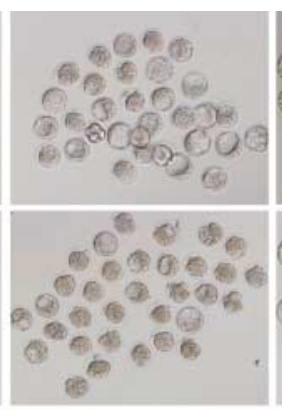

$\mathrm{D}$

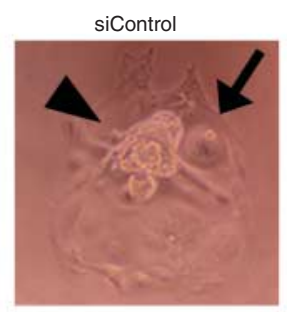

E4.5

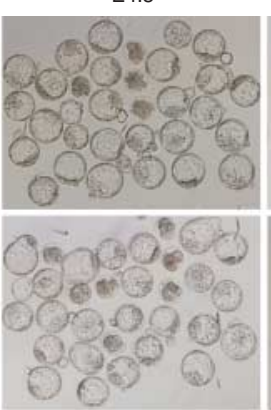

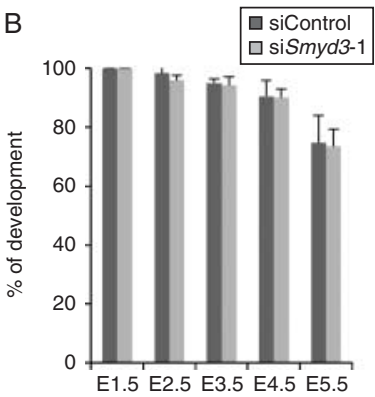

C

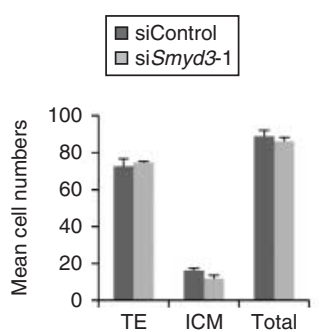

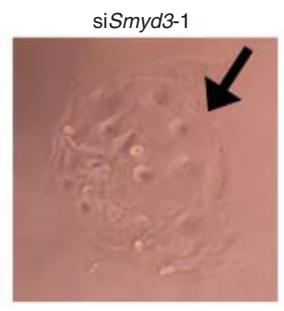

E5.5

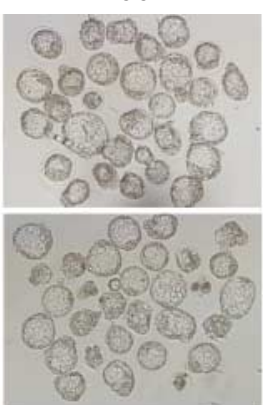

E

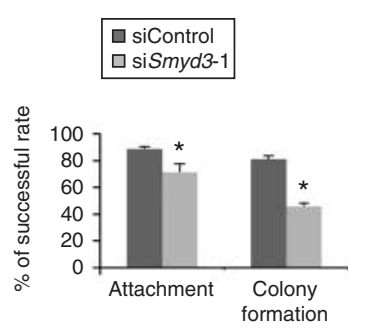

Figure 3 The effects of Smyd3 knockdown on pre- and post-implantation development. (A) Pairs of representative photos showing the development of preimplantation embryos injected with either siSmyd3-1 or siControl. Embryos were photographed at $36 \mathrm{~h}$ after IVF and at $24 \mathrm{~h}$ intervals thereafter. (B) The percentage of development at E1.5 ( $\geq$ two-cell), E2.5 ( $\geq$ four-cell), E3.5 ( $\geq$ morula), E4.5 ( $\geq$ blastocyst), and E5.5 ( $\geq$ hatching) in Smyd3knockdown and control embryos. Data are expressed as means \pm s.E.M. $(n=3)$. Twenty to 25 embryos were used in each experiment $(63$ and 69 embryos in total in siControl and siSmyd3-1 respectively). (C) The numbers of ICM and TE cells were assessed by counting OCT4-positive cells and CDX2-positive cells respectively. Total embryonic cell numbers were obtained by combining the numbers of ICM and TE cells. Data are expressed as means \pm S.E.M. $(n=16)$. (D) Photographs of the representative results of outgrowth experiments for control and Smyd3-knockdown embryos (arrowhead, ICM-derived colony; arrow, trophectoderm cells). (E) The successful percentages of attachment and ICM-derived colony formation in Smyd3-knockdown and control embryos after 4 days in culture $\left({ }^{*} P<0.05\right)$. Data are expressed as means \pm s.E.M. $(n=6)$. Thirteen to 83 embryos were used in each experiment (238 and 243 embryos in total in siControl and siSmyd3-1 respectively).

\section{Knockdown of Smyd3 does not induce apoptosis during peri-implantation development}

Because it has been reported that Smyd3 knockdown induces apoptosis in human cancer cells (Chen et al. 2007), we investigated the effects of Smyd3 knockdown on apoptosis in mouse peri-implantation embryos. However, there was no difference in the number of TUNEL-positive cells between Smyd3-knockdown and control embryos (Fig. 6).

\section{Discussion}

SMYD3 methylates both H3K4 and H4K5 (Hamamoto et al. 2004, Van Aller et al. 2012), recruits RNA polymerase II through an RNA helicase to form a transcription complex, and elicits its oncogenic effects by activating the transcription of downstream target genes (Hamamoto et al. 2004, 2006, Liu et al. 2007, 2013). SMYD3 is also involved in apoptosis and the inhibition of cell growth, migration, and invasion ( $\mathrm{Xu}$ et al. 2006, Zou et al. 2009). In the present paper, we observed Smyd3 mRNA expression patterns and protein localization during mouse preimplantation development and showed that Smyd3 knockdown led to a defect in the ability of Smyd3 mRNA to attach to a matrix and outgrowth in vitro and to a reduction in the number of viable offspring, which suggests that SMYD3 plays important roles during peri-implantation development.

Previous reports have shown that Oct4 and Sox2 form an Oct4/Sox2 complex and bind directly to their own

Table 1 Effect of Smyd3 knockdown on mouse embryo development.

\begin{tabular}{|c|c|c|c|c|c|}
\hline siRNA injected & $\begin{array}{c}\text { No. of trials (number } \\
\text { of recipients) }\end{array}$ & $\begin{array}{l}\text { No. of embryos } \\
\text { transferred }\end{array}$ & $\begin{array}{c}\text { No. of } \\
\text { pregnant mice }\end{array}$ & $\begin{array}{l}\text { No. of live offspring } \\
(\text { mean } \pm \text { s.E.M. })\end{array}$ & $\begin{array}{l}\text { Percentage of } \\
\text { live offspring }\end{array}$ \\
\hline siControl & 4 & $60^{\mathrm{a}}$ & 4 & $31(7.8 \pm 0.25)$ & $51.7^{*}$ \\
\hline siSmyd3-1 & 4 & $60^{\mathrm{a}}$ & 4 & $12(3.0 \pm 0.41)$ & $20.0^{\dagger}$ \\
\hline
\end{tabular}

${ }^{*}{ }^{+} P<0.05$.

${ }^{\mathrm{a}}$ Fifteen embryos were transferred to each recipient at the trial. 

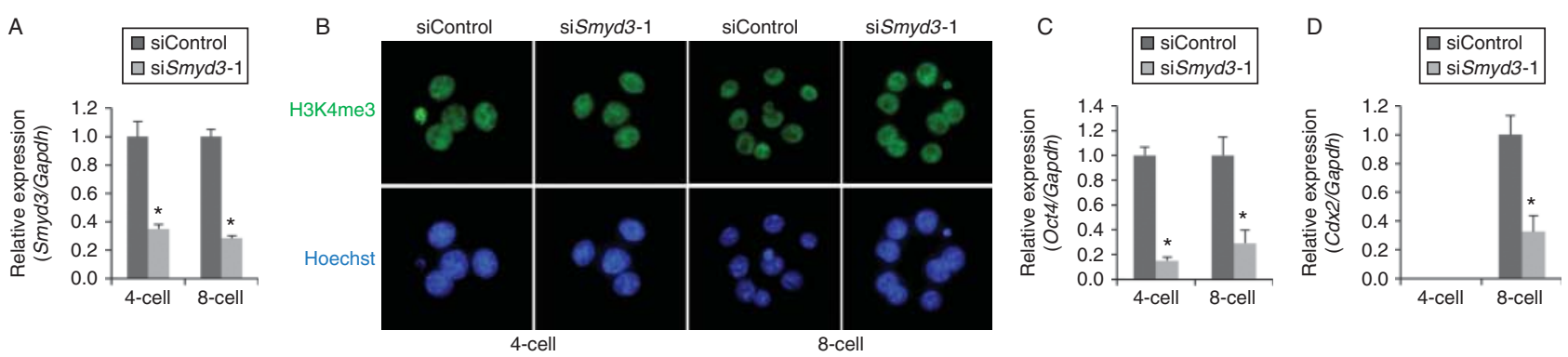

Figure 4 SMYD3 regulates the expression of Oct4 and Cdx2 at MGA. (A) qRT-PCR analysis of Smyd3 mRNA in Smyd3-knockdown and control embryos at the four- and eight-cell stages $\left({ }^{*} P<0.05\right)$. Expression levels were normalized to Gapdh as an internal control. Data are expressed as means \pm s.E.M. $(n=3)$. (B) Immunostaining of H3K4me3 in Smyd3-knockdown and control embryos at the four- and eight-cell stages (red, SMYD3; green, H3K4me3; blue, chromatin). (C and D) qRT-PCR analysis of Oct4 mRNA and Cdx2 mRNA in Smyd3-knockdown and control embryos at the four- and eight-cell stages $\left({ }^{*} P<0.05\right)$. Data are expressed as means \pm s.E.M. $(n=3)$.

promoter regions in embryonic stem (ES) cells (Chew et al. 2005, Okumura-Nakanishi et al. 2005). Oct4, Nanog, and Sox2 are reported to be regulated via the Oct4/Sox2 complex and to form a self-reinforcing regulatory loop in ES cells (Mitsui et al. 2003, Chew et al. 2005, Okumura-Nakanishi et al. 2005, Rodda et al. 2005). In mouse preimplantation embryos, Oct4, Nanog, and Sox2 are required for the maintenance of ICM pluripotency (Nichols et al. 1998, Avilion et al. 2003, Mitsui et al. 2003). Additionally, Nanog is known to negatively interact with Gata6, and both genes are known to be key regulators in the establishment of EPI and PE fates respectively (Morris et al. 2010,
Frankenberg et al. 2011, Kang et al. 2013, Schrode et al. 2014). Nanog-deficient mouse embryos are arrested during post-implantation development because of the widespread expression of Gata6 in the EPI (Mitsui et al. 2003, Frankenberg et al. 2011). By contrast, Gata6-deficient mouse embryos are arrested during postimplantation development because of the widespread expression of Nanog in the PE (Morris et al. 2010, Schrode et al. 2014). In the present study, we showed that Smyd3 knockdown led to the suppression of the embryonic transcription of Oct4 during the four-cell stage. In addition, we also showed that in blastocysts, Smyd3 knockdown abrogates the transcription of other
A

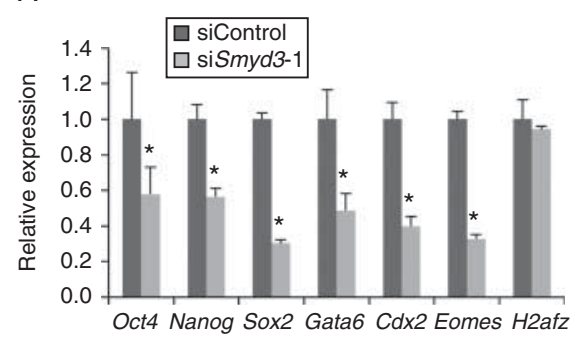

B
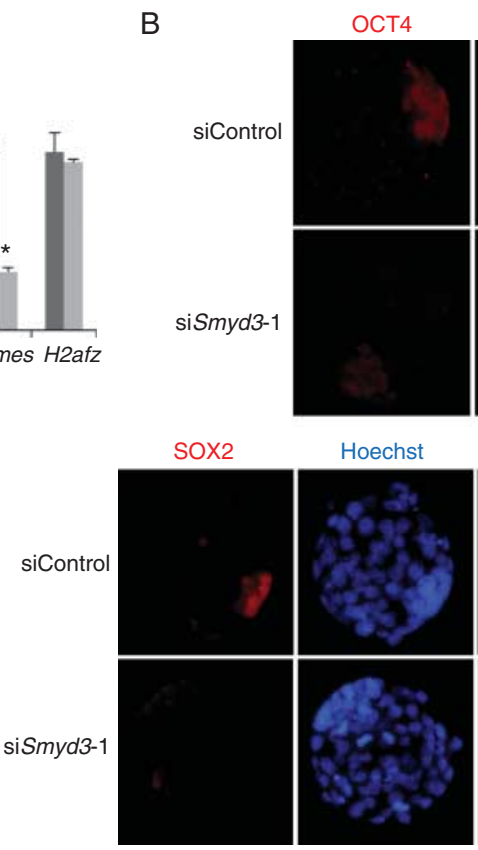

Hoechst

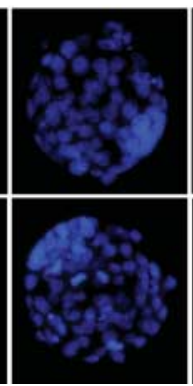

CDX2

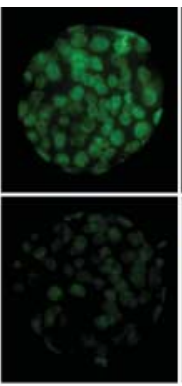

GATA6

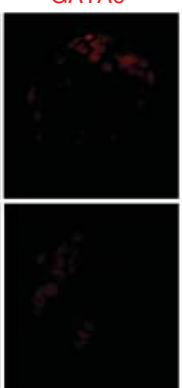

Hoechst

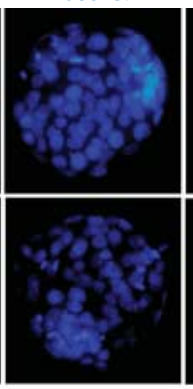

Hoechst

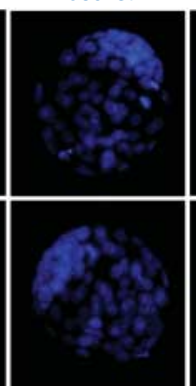

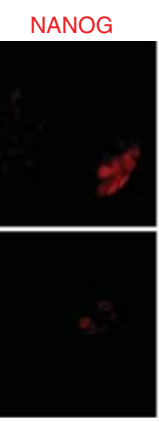

EOMES

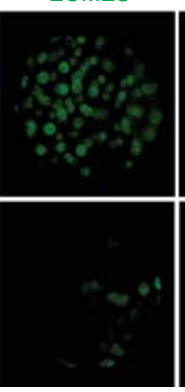

Hoechst

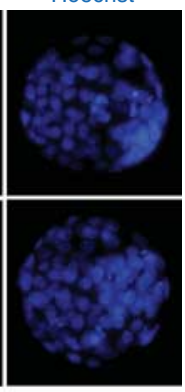

Hoechst

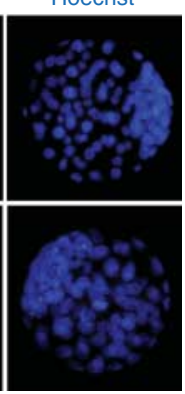

Figure 5 The effects of Smyd3 knockdown on the expression of lineage-specific genes in blastocysts. (A) qRT-PCR analysis of the early lineage markers Oct4, Nanog, Sox2, Cdx2, Eomes, Gata6, and H2afz in Smyd3-knockdown and control blastocyst embryos $\left({ }^{*} P<0.05\right)$. The expression levels were normalized using Gapdh as an internal control. Data are expressed as means \pm s.E.M. $(n=3)$. (B) Immunostaining of OCT4, CDX2, NANOG, SOX2, GATA6, and EOMES in Smyd3-knockdown and control blastocyst embryos (red, OCT4; green, CDX2; red, NANOG; red, SOX2; red, GATA6; green, EOMES; blue, chromatin). 

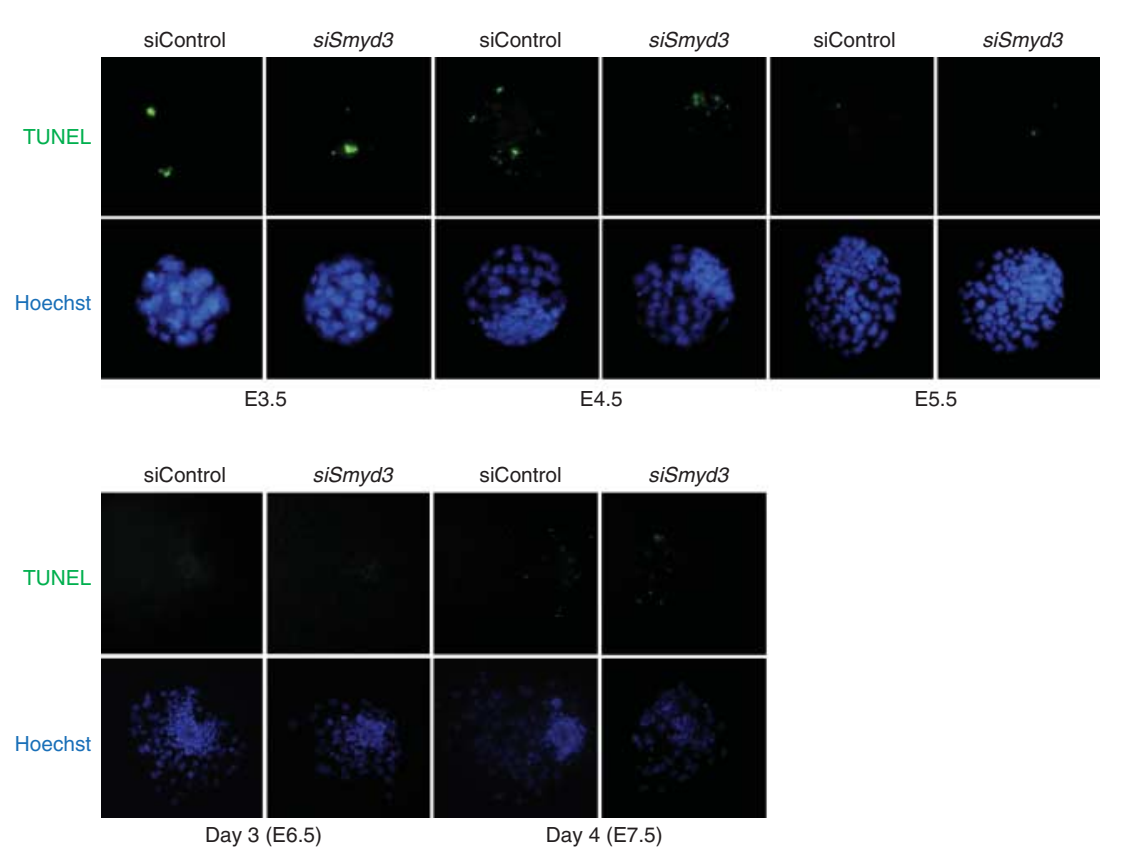

Figure 6 The effects of Smyd3 knockdown on the induction of apoptosis. TUNEL-positive cells (green nuclei) were detected. Photographs depict representative results of TUNEL assay for control and Smyd3-knockdown embryos before (E3.5-E5.5) and after (E6.5-E7.5) outgrowth. Nuclei are stained with Hoechst dye (blue).
ICM and EPI markers, such as Nanog and Sox2, and of PE markers, such as Gata6. Therefore, it is possible that the lack of pluripotency genes, such as Oct4, Nanog, and Sox2, and of PE-specific genes, such as Gata6, could account for the defects observed in Smyd3-knockdown embryos, including poor outgrowth in vitro and a reduction in the number of viable offspring. Furthermore, $C d \times 2$ and Eomes have been reported to be essential for the specification and differentiation of TE (Russ et al. 2000, Strumpf et al. 2005). Cdx2-knockout embryos fail to hatch from the zona pellucida or to implant in vivo, and they also fail to attach to matrix substrates in vitro, even when the zona pellucida has been removed (Strumpf et al. 2005). In the present study, we showed that Smyd3 knockdown led to the suppression of the embryonic transcription of $C d x 2$ during the eight-cell stages. Additionally, we also showed that in blastocysts, Smyd3 knockdown abrogates the transcription of TE markers, such as Eomes. Therefore, it is possible that the suppression of TE-specific genes, such as $C d x 2$ and Eomes, could account for a defect in the ability of Smyd3-knockdown embryos to attach to a matrix substrate in vitro. Furthermore, it has been reported that Smyd3 knockdown induces apoptosis in cancer (Chen et al. 2007). In the present study, however, we showed that the number of apoptotic cells was not increased in Smyd3-knockdown embryos. Together, these observations also suggest that SMYD3 plays an important role in peri-implantation embryonic development via the activation of lineage-specific gene expression. However, we demonstrated that in Smyd3knockdown embryos, global H3K4me3 levels appeared to be unchanged and that developmental arrest did not occur up to the blastocyst stage, even though Smyd3 knockdown suppressed the nuclear localization of SMYD3 protein at ZGA and MGA. Smyd3 trimethylates $\mathrm{H} 3 \mathrm{~K} 4$, and some chromatin remodeling complexes are recruited to H3K4me3 (He et al. 2005, Sims et al. 2005). These chromatin remodeling factors are involved in an open chromatin state, which correlates with a globally permissive transcriptional state. Within this 'loose' chromatin state, transcription factors can bind to the promoter regions of genes and activate transcription. The decreased expression of lineage-specific genes, such as Oct4, Nanog, and $C d x 2$, that we observed in the present study echoes results that have been obtained in Klf5-null embryos (Lin et al. 2010) and in Hmgpi-knockdown embryos (Yamada et al. 2010), which suggests that SMYD3 functions via these transcription factors to determine lineage specificity. Previous studies have shown that MLL2, one of the H3K4 methyltransferases, affects the global H3K4me3 levels at ZGA (AndreuVieyra et al. 2010), and the SETD1A/SETD1B methyltransferase complex that modifies $\mathrm{H} 3 \mathrm{~K} 4$ affects the global H3K4me3 levels at MGA (Bi et al. 2011). Additionally, previous studies have demonstrated that a knockdown of SMYD3 expression leads to a selective decrease in $\mathrm{H} 3 \mathrm{~K} 4$ methylation levels on oncogene promoter regions in cancer cells (Cock-Rada et al. 2012, Medjkane et al. 2012, Liu et al. 2013). From these observations, it is possible that the H3K4me3 level depends on the type of methyltransferase and that SMYD3 modifies H3K4 within the promoter regions of the lineage-specific genes. In addition, a recent report 
demonstrated that although global levels of H3K4me3 do not change upon the loss of Smyd3 in the human breast carcinoma cell line, MCF7, the global levels of H4K5me, a novel chromatin target of Smyd3, do change (Van Aller et al. 2012). This result indicates that SMYD3 is required for $\mathrm{H} 4 \mathrm{~K} 5$ methylation in cancer cells. It is also possible that SMYD3 is involved in the regulation of genes in mouse preimplantation embryos through the methylation of $\mathrm{H} 4 \mathrm{~K} 5$.

In summary, the present results demonstrated that Smyd3 knockdown does not have a critical effect on early embryonic development or on global H3K4me3 levels, but it does play an important role in early embryonic lineage commitment and peri-implantation development by regulating the expression of Oct4 and $C d x 2$ at MGA. Accordingly, we demonstrated the importance of Smyd3 as a key regulator of lineagespecific genes in mouse preimplantation embryos.

\section{Supplementary data}

This is linked to the online version of the paper at http://dx.doi. org/10.1530/REP-15-0019.

\section{Declaration of interest}

The authors declare that there is no conflict of interest that could be perceived as prejudicing the impartiality of the research reported.

\section{Funding}

This work was supported by a Grant-in-Aid for Scientific Research from the Japan Society for the Promotion of Science (grant number 23380164 to $\mathrm{N}$ Minami).

\section{References}

Akiyama T, Suzuki O, Matsuda J \& Aoki F 2011 Dynamic replacement of histone $\mathrm{H} 3$ variants reprograms epigenetic marks in early mouse embryos. PLoS Genetics 7 e1002279. (doi:10.1371/journal.pgen. 1002279)

Andreu-Vieyra CV, Chen R, Agno JE, Glaser S, Anastassiadis K, Stewart AF \& Matzuk MM 2010 MLL2 is required in oocytes for bulk histone 3 lysine 4 trimethylation and transcriptional silencing. PLoS Biology 8 e1000453. (doi:10.1371/journal.pbio.1000453)

Avilion AA, Nicolis SK, Pevny LH, Perez L, Vivian N \& Lovell-Badge R 2003 Multipotent cell lineages in early mouse development depend on SOX2 function. Genes and Development 17 126-140. (doi:10.1101/gad. 224503)

Bi Y, Lv Z, Wang Y, Hai T, Huo R, Zhou Z, Zhou Q \& Sha J 2011 WDR82, a key epigenetics-related factor, plays a crucial role in normal early embryonic development in mice. Biology of Reproduction 84 756-764. (doi:10.1095/biolreprod.110.084343)

Chazaud C, Yamanaka Y, Pawson T \& Rossant J 2006 Early lineage segregation between epiblast and primitive endoderm in mouse blastocysts through the Grb2-MAPK pathway. Developmental Cell 10 615-624. (doi:10.1016/j.devcel.2006.02.020)
Chen LB, Xu JY, Yang Z \& Wang GB 2007 Silencing SMYD3 in hepatoma demethylates RIZI promoter induces apoptosis and inhibits cell proliferation and migration. World Journal of Gastroenterology 13 5718-5724. (doi:10.3748/wjg.v13.i43.5718)

Chew JL, Loh YH, Zhang W, Chen X, Tam WL, Yeap LS, Li P, Ang YS, Lim B, Robson P et al. 2005 Reciprocal transcriptional regulation of Pou5f1 and Sox 2 via the Oct $4 /$ Sox 2 complex in embryonic stem cells. Molecular and Cellular Biology 25 6031-6046. (doi:10.1128/MCB.25.14.6031-6046. 2005)

Cock-Rada AM, Medjkane S, Janski N, Yousfi N, Perichon M, Chaussepied M, Chluba J, Langsley G \& Weitzman JB 2012 SMYD3 promotes cancer invasion by epigenetic upregulation of the metalloproteinase MMP-9. Cancer Research 72 810-820. (doi:10.1158/0008-5472. CAN-11-1052)

Feng Q, Wang $\mathrm{H}, \mathrm{Ng} \mathrm{HH}$, Erdjument-Bromage $\mathrm{H}$, Tempst $\mathrm{P}$, Struhl K \& Zhang Y 2002 Methylation of H3-lysine 79 is mediated by a new family of HMTases without a SET domain. Current Biology 12 1052-1058. (doi:10.1016/S0960-9822(02)00901-6)

Frankenberg S, Gerbe F, Bessonnard S, Belville C, Pouchin P, Bardot O \& Chazaud C 2011 Primitive endoderm differentiates via a three-step mechanism involving Nanog and RTK signaling. Developmental Cell 21 1005-1013. (doi:10.1016/j.devcel.2011.10.019)

Fujii T, Tsunesumi S, Yamaguchi K, Watanabe S \& Furukawa Y 2011 Smyd3 is required for the development of cardiac and skeletal muscle in zebrafish. PLOS ONE 6 e23491. (doi:10.1371/journal.pone.0023491)

Gustafsson MG 2005 Nonlinear structured-illumination microscopy: wide-field fluorescence imaging with theoretically unlimited resolution. PNAS 102 13081-13086. (doi:10.1073/pnas.0406877102)

Hamamoto R, Furukawa Y, Morita M, limura Y, Silva FP, Li M, Yagyu R \& Nakamura Y 2004 SMYD3 encodes a histone methyltransferase involved in the proliferation of cancer cells. Nature Cell Biology 6 731-740. (doi:10.1038/ncb1151)

Hamamoto R, Silva FP, Tsuge M, Nishidate T, Katagiri T, Nakamura Y \& Furukawa Y 2006 Enhanced SMYD3 expression is essential for the growth of breast cancer cells. Cancer Science 97 113-118. (doi:10.1111/ j.1349-7006.2006.00146.x)

Hamatani T, Carter MG, Sharov AA \& Ko MS 2004 Dynamics of global gene expression changes during mouse preimplantation development. Developmental Cell 6 117-131. (doi:10.1016/S1534-5807(03)00373-3)

He GHY, Helbing CC, Wagner MJ, Sensen CW \& Riabowol K 2005 Phylogenetic analysis of the ING family of PHD finger proteins. Molecular Biology and Evolution 22 104-116. (doi:10.1093/molbev/ msh256)

Hirasawa R, Chiba H, Kaneda M, Tajima S, Li E, Jaenisch R \& Sasaki H 2008 Maternal and zygotic Dnmt1 are necessary and sufficient for the maintenance of DNA methylation imprints during preimplantation development. Genes and Development 22 1607-1616. (doi:10.1101/ gad.1667008)

Hosny NA, Song M, Connelly JT, Ameer-Beg S, Knight MM \& Wheeler AP 2013 Super-resolution imaging strategies for cell biologists using a spinning disk microscope. PLOS ONE 8 e74604. (doi:10.1371/journal. pone.0074604)

Isaji Y, Murata M, Takaguchi N, Mukai T, Tajima Y, Imai H \& Yamada M 2013 Valproic acid treatment from the 4-cell stage improves Oct4 expression and nuclear distribution of histone H3K27me3 in mouse cloned blastocysts. Journal of Reproduction and Development 59 196-204. (doi:10.1262/jrd.2012-156)

Jeong YJ, Choi HW, Shin HS, Cui XS, Kim NH, Gerton GL \& Jun JH 2005 Optimization of real time RT-PCR methods for the analysis of gene expression in mouse eggs and preimplantation embryos. Molecular Reproduction and Development 71 284-289. (doi:10.1002/mrd.20269)

Kang M, Piliszek A, Artus J \& Hadjantonakis AK 2013 FGF4 is required for lineage restriction and salt-and-pepper distribution of primitive endoderm factors but not their initial expression in the mouse. Development 140 267-279. (doi:10.1242/dev.084996)

Latham KE \& Schultz RM 2001 Embryonic genome activation. Frontiers in Bioscience 6 D748-D759. (doi:10.2741/Latham)

Lepikhov K \& Walter J 2004 Differential dynamics of histone H3 methylation at positions $\mathrm{K} 4$ and $\mathrm{K} 9$ in the mouse zygote. $B M C$ Developmental Biology 4 12. (doi:10.1186/1471-213X-4-12)

Li L, Zheng P \& Dean J 2010 Maternal control of early mouse development. Development 137 859-870. (doi:10.1242/dev.039487) 
Lin SC, Wani MA, Whitsett JA \& Wells JM 2010 Klf5 regulates lineage formation in the pre-implantation mouse embryo. Development 137 3953-3963. (doi:10.1242/dev.054775)

Liu C, Fang X, Ge Z, Jalink M, Kyo S, Björkholm M, Gruber A, Sjöberg J \& Xu D 2007 The telomerase reverse transcriptase (hTERT) gene is a direct target of the histone methyltransferase SMYD3. Cancer Research 67 2626-2631. (doi:10.1158/0008-5472.CAN-06-4126)

Liu C, Wang C, Wang K, Liu L, Shen Q, Yan K, Sun X, Chen J, Liu J, Ren H et al. 2013 SMYD3 as an oncogenic driver in prostate cancer by stimulation of androgen receptor transcription. Journal of the National Cancer Institute 105 1719-1728. (doi:10.1093/jnci/djt304)

Livak KJ \& Schmittgen TD 2001 Analysis of relative gene expression data using real-time quantitative PCR and the $2(-\Delta \Delta C(\mathrm{~T}))$ Method. Methods 25 402-408. (doi:10.1006/meth.2001.1262)

Mamo S, Gal AB, Bodo S \& Dinnyes A 2007 Quantitative evaluation and selection of reference genes in mouse oocytes and embryos cultured in vivo and in vitro. BMC Developmental Biology 7 14. (doi:10.1186/ 1471-213X-7-14)

Medjkane S, Cock-Rada A \& Weitzman JB 2012 Role of the SMYD3 histone methyltransferase in tumorigenesis: local or global effects? Cell Cycle $\mathbf{1 1}$ 1865. (doi:10.4161/cc.20415)

Mitsui K, Tokuzawa Y, Itoh H, Segawa K, Murakami M, Takahashi K, Maruyama M, Maeda M \& Yamanaka S 2003 The homeoprotein Nanog is required for maintenance of pluripotency in mouse epiblast and ES cells. Cell 113 631-642. (doi:10.1016/S0092-8674(03)00393-3)

Morris SA, Teo RT, Li H, Robson P, Glover DM \& Zernicka-Goetz M 2010 Origin and formation of the first two distinct cell types of the inner cell mass in the mouse embryo. PNAS 107 6364-6369. (doi:10.1073/pnas. 0915063107)

Nichols J, Zevnik B, Anastassiadis K, Niwa H, Klewe-Nebenius D, Chambers I, Scholer H \& Smith A 1998 Formation of pluripotent stem cells in the mammalian embryo depends on the POU transcription factor Oct4. Cell 95 379-391. (doi:10.1016/S0092-8674(00)81769-9)

Nowak SJ \& Corces VG 2004 Phosphorylation of histone H3: a balancing act between chromosome condensation and transcriptional activation. Trends in Genetics 20 214-220. (doi:10.1016/j.tig.2004.02.007)

Okumura-Nakanishi S, Saito M, Niwa H \& Ishikawa F 2005 Oct-3/4 and Sox2 regulate Oct-3/4 gene in embryonic stem cells. Journal of Biological Chemistry 280 5307-5317. (doi:10.1074/jbc.M410015200)

Ralston A \& Rossant J 2008 Cdx2 acts downstream of cell polarization to cell-autonomously promote trophectoderm fate in the early mouse embryo. Developmental Biology 313 614-629. (doi:10.1016/j.ydbio. 2007.10.054)

Rodda DJ, Chew JL, Lim LH, Loh YH, Wang B, Ng HH \& Robson P 2005 Transcriptional regulation of nanog by OCT4 and SOX2. Journal of Biological Chemistry 280 24731-24737. (doi:10.1074/jbc.M502573200)

Rossant J 2004 Lineage development and polar asymmetries in the periimplantation mouse blastocyst. Seminars in Cell \& Developmental Biology 15 573-581. (doi:10.1016/j.semcdb.2004.04.003)

Russ AP, Wattler S, Colledge WH, Aparicio SA, Carlton MB, Pearce JJ, Barton SC, Surani MA, Ryan K, Nehls MC et al. 2000 Eomesodermin is required for mouse trophoblast development and mesoderm formation. Nature 404 95-99. (doi:10.1038/35003601)

Santos F, Peters AH, Otte AP, Reik W \& Dean W 2005 Dynamic chromatin modifications characterise the first cell cycle in mouse embryos. Developmental Biology 280 225-236. (doi:10.1016/j.ydbio.2005.01.025)

Sarmento OF, Digilio LC, Wang Y, Perlin J, Herr JC, Allis CD \& Coonrod SA 2004 Dynamic alterations of specific histone modifications during early murine development. Journal of Cell Science 117 4449-4459. (doi:10. 1242/jcs.01328)
Schrode N, Saiz N, Di Talia S \& Hadjantonakis AK 2014 GATA6 levels modulate primitive endoderm cell fate choice and timing in the mouse blastocyst. Developmental Cell 29 454-467. (doi:10.1016/j.devcel. 2014.04.011)

Schultz RM \& Worrad DM 1995 Role of chromatin structure in zygotic gene activation in the mammalian embryo. Seminars in Cell Biology 6 201-208. (doi:10.1006/scel.1995.0028)

Shilatifard A 2006 Chromatin modifications by methylation and ubiquitination: implications in the regulation of gene expression. Annual Review of Biochemistry 75 243-269. (doi:10.1146/annurev.biochem.75.103004. 142422)

Sims RJ III, Chen CF, Santos-Rosa H, Kouzarides T, Patel SS \& Reinberg D 2005 Human but not yeast CHD1 binds directly and selectively to histone $\mathrm{H} 3$ methylated at lysine 4 via its tandem chromodomains. Journal of Biological Chemistry 280 41789-41792. (doi:10.1074/ jbc.C500395200)

Sterner DE \& Berger SL 2000 Acetylation of histones and transcriptionrelated factors. Microbiology and Molecular Biology Reviews 64 435-459. (doi:10.1128/MMBR.64.2.435-459.2000)

Strumpf D, Mao CA, Yamanaka Y, Ralston A, Chawengsaksophak K, Beck F \& Rossant J 2005 Cdx2 is required for correct cell fate specification and differentiation of trophectoderm in the mouse blastocyst. Development 132 2093-2102. (doi:10.1242/dev.01801)

Suzuki S, Nozawa Y, Tsukamoto S, Kaneko T, Imai H \& Minami N 2013 ING3 is essential for asymmetric cell division during mouse oocyte maturation. PLOS ONE 8 e74749. (doi:10.1371/journal.pone.0074749)

Suzuki S, Tsukiyama T, Kaneko T, Imai H \& Minami N 2015 A hyperactive piggyBac transposon system is an easy-to-implement method for introducing foreign genes into mouse preimplantation embryos. Journal of Reproduction and Development. In press. (doi:10.1262/jrd.2014-157)

Tsukamoto S, Hara T, Yamamoto A, Ohta Y, Wada A, Ishida Y, Kito S, Nishikawa T, Minami N, Sato K et al. 2013 Functional analysis of lysosomes during mouse preimplantation embryo development. Journal of Reproduction and Development 59 33-39.

Van Aller GS, Reynoird N, Barbash O, Huddleston M, Liu S, Zmoos AF, McDevitt P, Sinnamon R, Le B, Mas G et al. 2012 Smyd3 regulates cancer cell phenotypes and catalyzes histone $\mathrm{H} 4$ lysine 5 methylation. Epigenetics 7 340-343. (doi:10.4161/epi.19506)

Xu JY, Chen LB, Yang Z, Wei HY \& Xu RH 2006 Inhibition of SMYD3 gene expression by RNA interference induces apoptosis in human hepatocellular carcinoma cell line HepG2. Ai Zheng 25 526-532.

Yamada M, Hamatani T, Akutsu H, Chikazawa N, Kuji N, Yoshimura Y \& Umezawa A 2010 Involvement of a novel preimplantation-specific gene encoding the high mobility group box protein Hmgpi in early embryonic development. Human Molecular Genetics 19 480-493. (doi:10.1093/ hmg/ddp512)

Zhang Y \& Reinberg D 2001 Transcription regulation by histone methylation: interplay between different covalent modifications of the core histone tails. Genes and Development 15 2343-2360. (doi:10.1101/gad.927301)

Zou JN, Wang SZ, Yang JS, Luo XG, Xie JH \& Xi T 2009 Knockdown of SMYD3 by RNA interference down-regulates c-Met expression and inhibits cells migration and invasion induced by HGF. Cancer Letters 280 78-85. (doi:10.1016/j.canlet.2009.02.015)

Received 13 January 2015

First decision 9 February 2015

Revised manuscript received 2 April 2015

Accepted 24 April 2015 